-Journal Publications • Research Consultancy

\title{
APPROACHES TO IMAGE PROCESSING USING THE TOOLS OF FUZZY SETS
}

\section{R. KH. KHAMDAMOV ${ }^{1} \&$ E. A. SALIYEV ${ }^{2}$}

${ }^{l}$ Doctor of Technical Sciences, prof.Director of the Scientific and Innovation Center of Information and Communication Technologies at the Tashkent University of Information Technologies

${ }^{2}$ Research., Associate Professor. The competitor of the Scientific and Innovation Center of Information and Communication Technologies at the Tashkent University of Information Technologies.

ABSTRACT
In this paper, an approach to processing images by applying the concept of fuzzy sets is considered for the
purpose of improving the quality of images of image segmentation and contour extraction in images. Studied method of
image segmentation that use the algorithms of fuzzy clustering
KEYWORDS: Fuzzy Sets, Image Segmentation \& Fuzzy Clustering

Received: Jul 20, 2017; Accepted: Aug 09, 2017; Published: Dec 29, 2017; Paper Id.: IJCSEITRFEB20181

\section{INTRODUCTION}

In recent years in developed countries of the world conduct studies on the use of fuzzy techniques in image processing, which is associated with the following: 1) these methods are powerful tools for the representation and processing of knowledge; 2) they can manage the uncertainty and ambiguity efficiently. In many applications of image processing required to use expert knowledge to overcome some of the difficulties (e.g. Object recognition, scene analysis). In fuzzy set theory and fuzzy logic are powerful tools to represent and process human knowledge in the form of fuzzy IF-THEN rules. On the other hand, many difficulties in image processing arise because of the randomness, ambiguity and uncertainty in the data used in this task. To work with accidents in the processing of the images can be used in probability theory for other kinds of imperfections, for example, geometric blurring can be present on the basis of fuzzy sets and fuzzy logic. On the basis of the goals and objectives of the research defined the architecture of image processing system based on the concept of fuzzy sets. A functional scheme of this system is shown in Figure 1.

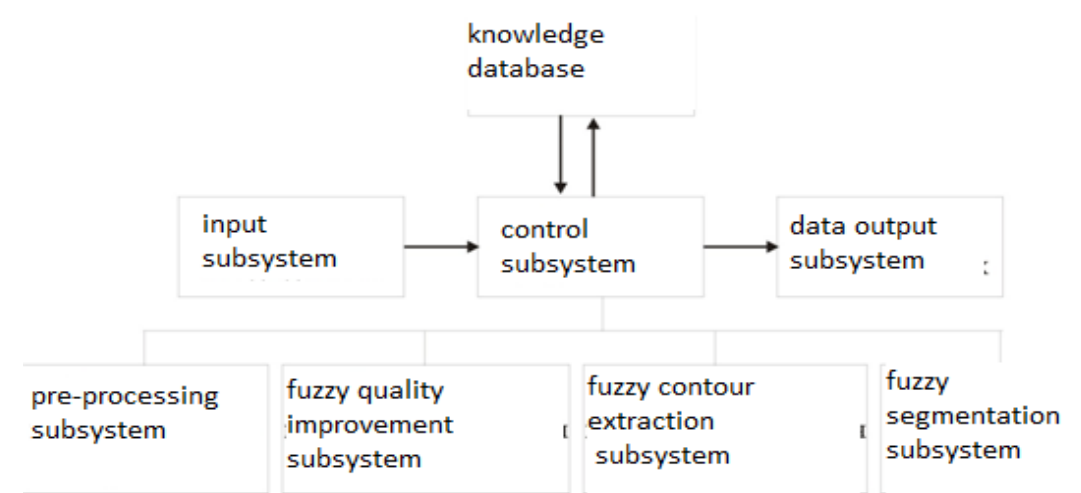

Figure 1: Functional Scheme of the Developed System

In this article the issue of imaging with the application of the concept of fuzzy sets is considered in relation 
to the following objectives: improving the quality of images, image segmentation and contour extraction on images.

Despite the progress made in the field of digital image processing using fuzzy sets, there are a number of outstanding tasks. Among them include the problem of adequate display subject region in a fuzzy system, selection of models, fuzzy logic and their integration into a single intelligent system.

Thus, issues of digital image processing using the tools of fuzzy sets are investigated insufficiently. Therefore, the development and improvement of methods of digital image processing based on fuzzy set theory, are very important.

\section{IMPROVING THE QUALITY OF THE IMAGES}

In [1] were among the first considered to improve quality by using fuzzy logic. It extraction method of fuzzy properties of grayscale images that can be used to enhance the contrast of this image. Improving the quality of the source image is usually one of the first steps in computer vision tasks. Methods of improving image quality, as a rule, allow you to remove noise, to smooth the regions where the gray levels did not significantly change, and highlight sharp changes in gray levels.

Since fuzzy logic allows including heuristic knowledge about its specific application in the form of rules, it is ideal to build the system of image improvement. This led to the development of various methods of image enhancement based on fuzzy logic. Next, a brief look at some of them.

In [2] proposes a dynamic filter to reduce the constriction of the range of values of the brightness and increasing contrast, using an approach based on fuzzy rules. The method is based on the algorithm given in [3]. In [4] proposed a nonlinear fuzzy filter for image processing. It is known that the averaging filter effectively removes Gaussian noise, and filters based on order statistics, such as median filter, are used effectively for the removal of impulse noise. To combine these two filters in [5] used fuzzy logic.

The following is the algorithm of linear increasing contrast with fuzzy initial information.

Image entered into the computer, often low contrast, i.e. they have a change of brightness is small compared to its mean value. The brightness are not changing from black to white and from gray to a brighter gray. That is the real brightness range is much less than acceptable (grayscale). The task of improving contrast is to "stretch" the range of brightness of the image on the entire scale.

The essence of feature-based image processing is as follows. Let $f(x, y)$ and $g(x, y)$ - the brightness values of the source and obtained after image processing, respectively, in frame with Cartesian coordinates $X-$ line number and $y$ - column number.

Element wise processing means that there is a functional dependence between the brightness

$$
g(x, y)=F(f(x, y))
$$

Allowing the value of the original signal to determine the value of the output signal.

The task of konstrastom is associated with improved matching of the dynamic range of the image and the screen on which you are rendering. If the digital representation of each reference image is assigned 1 byte ( 8 bits) memory 
devices, input or output signals can be one of 256 values. Typically, the worker uses the range $0 . .255$, with 0 representing when rendering the black level and the value 255 white level. Suppose that the minimum and maximum brightness of the original image are equal to $f_{\min }$ and $f_{\max }$ accordingly.

If these options are or one of them is significantly different from the boundary values of the luminance range, the rendered image looks like an uncomfortable, tiring during observation.

It is often useful to consider the image as a realization of a fuzzy random process. We introduce a generative image continuous random function $f(x, y)$ two variables o, ial coordinates $x, y$. Random process $f(x, y)$ fully described by the joint probability density $P[A]$.

\section{This Problem can be Solved with a Piecemeal Conversion of a Linear Contrast:}

$$
g(x, y)=a f(x, y)+b
$$

ie, are these $a$ and $b$, that lead the fuzzy values of the field brightness to some standard values. Here are preliminarily estimated $M[f(x, y)], \sigma[f(x, y)]$, and the odds $a$, bare selected so that for the output field get $M[\mathrm{~g}(x, y)], \sigma[\mathrm{g}(x, y)]$ :

$$
\begin{aligned}
& \bar{g}(x, y)=\frac{f(x, y)-M[f(x, y)]}{\sigma[f(x, y)]} \cdot \sigma[g(x, y)]+M[g(x, y)]= \\
& =\frac{\sigma[g(x, y)]}{\sigma[f(x, y)]} f(x, y)+M[g(x, y)]-M[f(x, y)] \frac{\sigma[g(x, y)]}{\sigma[f(x, y)]},
\end{aligned}
$$

i.e.

$$
a=\frac{\sigma[g(x, y)]}{\sigma[f(x, y)]} ; b=M[g(x, y)]-M[f(x, y)] \frac{\sigma[g(x, y)]}{\sigma[f(x, y)]} .
$$

Here

$$
\begin{aligned}
& M[f(x, y)]=\frac{\sum_{i=1}^{k} f_{i}(x, y) \cdot \mu_{i}^{f}(x, y)}{\sum_{i=1}^{k} \mu_{i}^{f}(x, y)}, M[g(x, y)]=\frac{\sum_{i=1}^{k} g_{i}(x, y) \cdot \mu_{i}^{g}(x, y)}{\sum_{i=1}^{k} \mu_{i}^{g}(x, y)} ; \\
& g(x, y)=F(f(x, y))= \begin{cases}0, & \bar{g}(x, y)<0, \\
\bar{g}(x, y), & 0 \leq \bar{g}(x, y) \leq 255, \\
255, & \bar{g}(x, y)>255 .\end{cases}
\end{aligned}
$$

Thus, at image processing is required for some signs to identify some homogeneous areas of the image. The stages of image pre-processing can reduce the influence of distortion on the recognition process. In Figure 2 shows the 
result of a linear increase in contrast with fuzzy initial information.
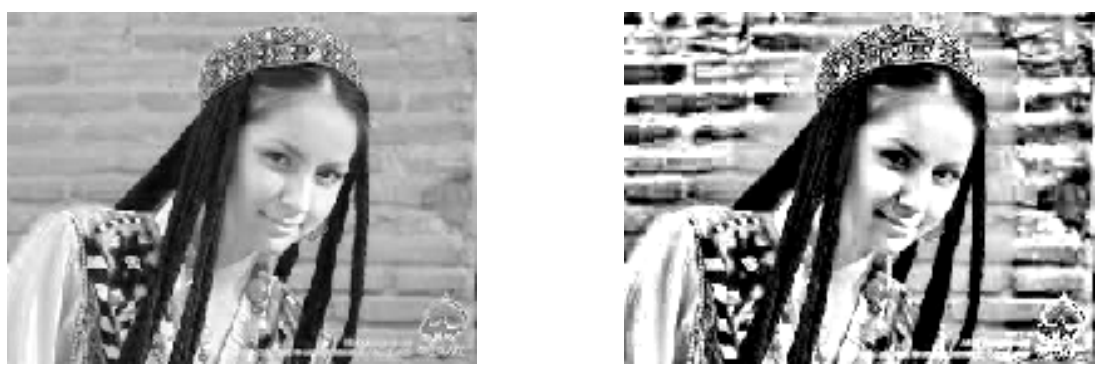

Figure 2: The Result of Linear Increasing in Contrast with Fuzzy Initial Information

\section{IMAGE SEGMENTATION}

Conceptual relationship between the segmentation and the theory of fuzzy sets is based on the fact that the structuring of complex images it is necessary to consider the fact that there are many real objects that do not have clear boundaries in nature. Requirement of the need to ensure uniqueness in segmentation and fuzzy data in this case is inadequate, especially when taking account of minor differences or for segments of complex shape, the overlapping between them.

On the basis of classical (crisp) methods of image segmentation is the determination of the values (the centroid) that characterize each segment in a given feature space and the classification of an object to a class based on some measure, usually a distance in the feature space.

Fuzzy or soft segmentation, introduces the notion of fuzzy segments and the membership function of pixels to

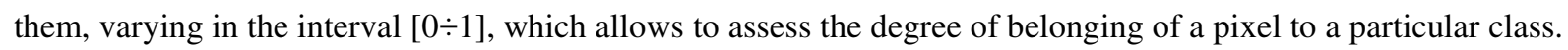

Methods of image segmentation based on fuzzy set theory are divided into the following groups.

\section{Threshold Segmentation}

Methods of this group are one of the simplest methods of image segmentation and divide the image into the object area and the background. Thus, these methods are based on comparison with a threshold to form a binary image in which the pixel value belonging to the objects is 1 , and the value of the pixels belonging to the background -0 . There are several traditional methods for the determination of thresholds, adequate original image [6,7]. To determine the optimal threshold, it is possible to calculate measures such as linear/quadratic index of fuzziness [8], fuzzy compactness [9] or the index of coverage [10]. Fuzzy deviation and probability measures can also be used to segment images into object and background [11].

\section{Segmentation Based on Clustering Techniques}

The first method of fuzzy clustering was the method of fuzzy C-means (Fuzzy C-means - FCM) [40, 45], which has currently a lot of modifications [12]. FCM method is based on the use of ideas and mathematical tools of fuzzy logic. In the FCM algorithm, each pixel in the image corresponds to a vector of membership functions to each class on the basis of which to draw conclusions about the nature of the object. 
Result of segmentation using the FCM algorithm depends strongly on the chosen measure. The Euclidean distance is effective only when the clusters are well-separated and approximately equal in size. Otherwise, it can be used by other algorithms such as the algorithm proposed in [13], or the algorithm of decomposition of the Gaussian mixture.

Let this real set $X=\left\{x_{1}, x_{2}, . ., x_{m}\right\} \subset R$ and k real numbers $s_{1}, s_{2}, . ., s_{k}$ in ascending order, namely:

$$
s_{1}<s_{2},<. .<s_{k}
$$

Fuzzy partitioning $A_{1}, A_{2}, \ldots, A_{k}$ for a set $\mathrm{X}$ can be organized using triangular functions.

When developing algorithm of fuzzy clustering for image segmentation, first, we consider the membership functions of:

$$
\mu_{1}(x)=\mu\left(x, s_{1} ; s_{2}\right)
$$

For $i=2,3, \ldots, k-1$

$$
\mu_{i}(x)=\mu\left(x, s_{i} ; s_{i-1}\right) \wedge \mu\left(x, s_{i} ; s_{i+1}\right), \mu_{k}(x)=\mu\left(x, s_{k} ; s_{k-1}\right)
$$

Functions $\mu_{1}, \mu_{2}, . ., \mu_{k}$ identify the partition split in the following equality:

$$
\mu_{1}+\mu_{2}+\ldots+\mu_{k}=1
$$

Secondly, determined the defuzzification operator $\tau(\mu, \gamma)$, which applies to a fuzzy partition:

$$
\tau(\mu, \gamma)=\left(\tau_{1}, \tau_{2}, . ., \tau_{k}\right)
$$

where

$$
\tau(x, \mu, \gamma)=\frac{\mu_{i}^{\gamma}(x)}{\sum_{j=1}^{k} \mu_{i}^{\gamma}(x)}
$$

Let $v_{i}$ and $F_{i}$ are defined as follows:

$$
v_{i}(x)=\mu_{i}(x) \wedge \tau_{i}(x, \mu ; \gamma), F_{i}\left(s_{i}\right)=\frac{\sum_{j=1}^{m} v_{i}\left(x_{j}\right) \cdot x_{j}}{\sum_{j=1}^{m} v_{i}\left(x_{j}\right)}
$$

Let us now consider the following restriction for the parameters $s_{1}, s_{2}, . ., S_{k}$ :

$$
s_{i}=F_{i}\left(s_{i}\right) .(1)
$$

Fuzzy set $A_{i}$ is determined by the equation (1). Center $s_{i}$ belongs to the convex hull of a set $\mathrm{X}$, and it is a fixed point for the function $F_{i}$. 
Results are obtained using the following fuzzy clustering algorithm.

Step 1: Initialized the number of clusters $\mathrm{k}$, defuzzification parameter $\gamma$, parameter stop procedure $\delta$, index of the iteration $l=0$ and cluster centers. Next, calculated fuzzy membership functions and functions d.zzification components $\tau_{1}^{(0)}, \tau_{2}^{(0)}, . ., \tau_{k}^{(0)}$

Step 2: Index of the iteration increased, i.e. $l \rightarrow l+1$. We calculate the centers of cluster $s_{1}^{(l)}=F_{1}\left(s_{1}^{(l-1)}\right), s_{2}^{(l)}=F_{2}\left(s_{2}^{(l-1)}\right), \ldots, s_{k}^{(l)}=F_{k}\left(s_{k}^{(l-1)}\right), \quad$ fuzzy $\quad$ membership $\quad$ functions $\mu_{1}^{(l)}, \mu_{2}^{(l)}, . ., \mu_{k}^{(l)}$, defuzzification components $\tau_{1}^{(l)}, \tau_{2}^{(l)}, . ., \tau_{k}^{(l)}$ and function $v_{1}^{(l)}, v_{2}^{(l)}, . ., v_{k}^{(l)}$.

Step 3: Calculate $d=\sum_{i=1}^{k}\left|\mu_{i}^{(l)}-\mu_{i}^{(l-1)}\right|$. If $d>\delta$, then return to step 2, otherwise go to step 4 .

Step 4: Saving data and end.

In Figure 3 shown results of the considered algorithms on different images.

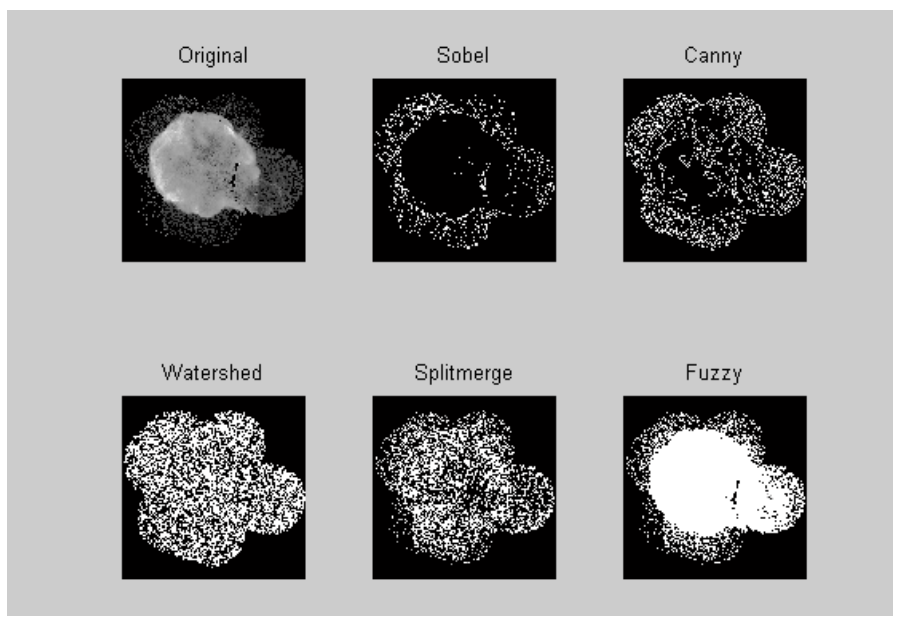

a)

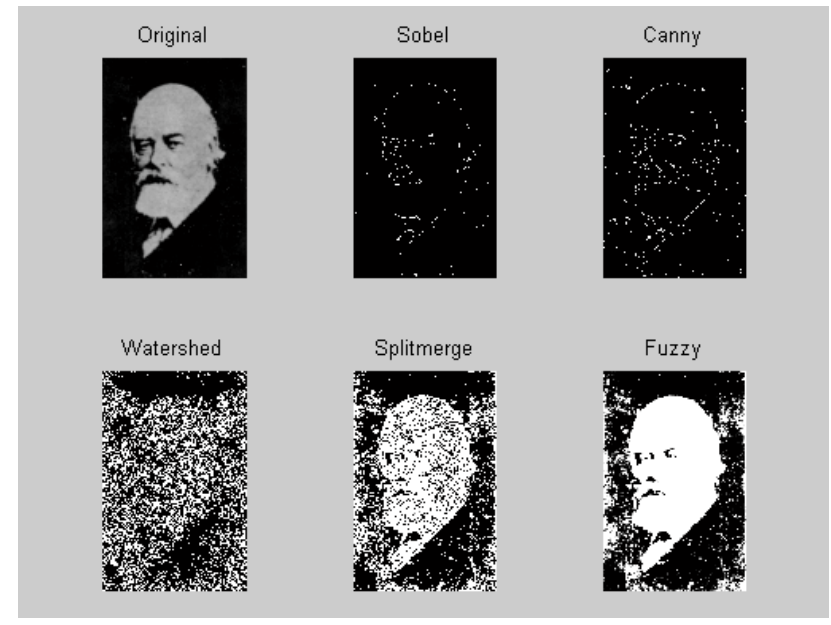

b)

Figure 3: The Results of the Algorithms Discussed in a) First , b)Second Images 


\section{ALLOCATION OF THE CIRCUITS}

Polygon selection is an important part of many computer vision system. Ideally, the contours correspond to the boundaries of objects, and therefore, the allocation of contours allows segmenting the image into meaningful regions. However, the term "circuit" is rather vague, heuristic, and even subjective concept. In [14] given the following definition of the contour: the contour point is a pixel in the neighborhood of which there is a significant local change of intensity; the contours are image fragments representing a set of points of the contour. As can be seen from this definition, there are several possibilities of fuzzification of the concept of "contour", because it involves two variables: spatial position and intensity. At present, there are several fuzzy models, which try to highlight the contours in the image, and this section discusses some of them.

In [15] described the method of allocation of contours, based on the FIRE (fuzzy inference ruled by else-action fuzzy conclusion on the basis of OTHERWISE-action) paradigm, which is relatively immune to noise. It uses the differences of the gray levels in a neighborhood of $3 \times 3$ as inputs in the fuzzy rules.

In [16] it is shown that using statistics such as range and variance of window intensities can be as effective as the use of traditional, such as the evaluation of gradients. In [17] proposes a method for contour extraction based on fuzzy logic, where local features such as gradient, symmetry, and straightness are combined in order to introduce the concept of "contour" and "angle". It argues that the traditional definition of contour points as points with high gradient between two uniform plasma regions is not valid in the corners (where the uniform region has an acute angle).

\section{Object Detection Based on Color}

There are a large number of tasks, one part of which is the question of automatic recognition of persons in images. Approaches to this question are many, but this material we will consider a method of automatic face detection in images based on color analysis.

Read a source image (Figure 4 - 7).

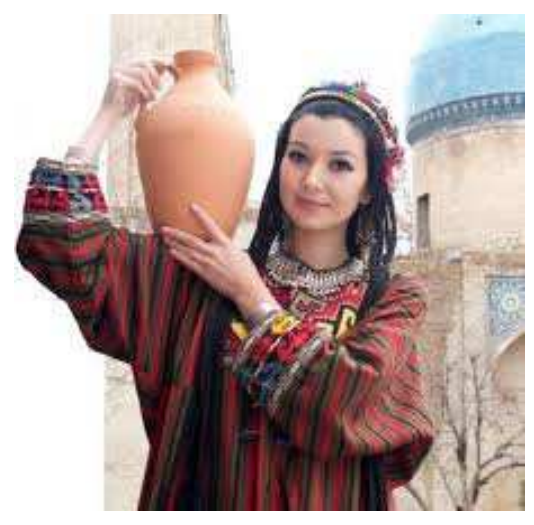

Figure 4: 1-Th Original Image

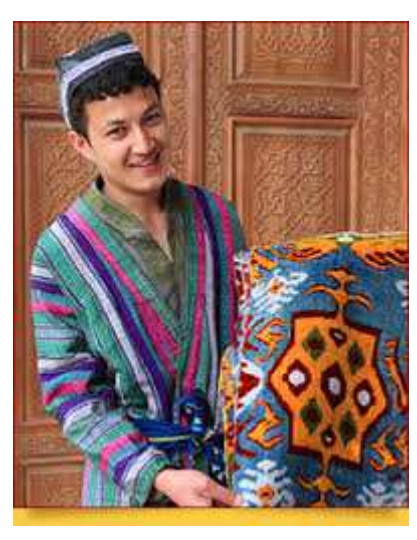

Figure 5: 2nd Source Image 


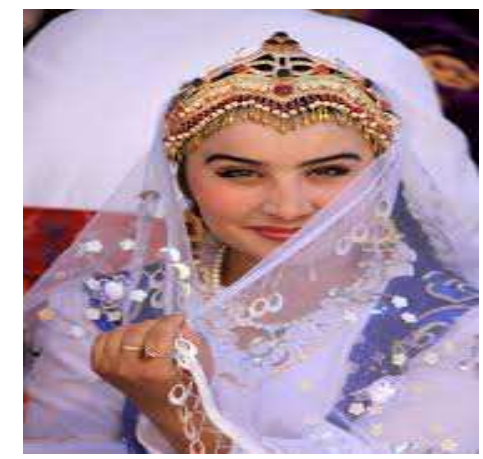

Figure 6: 3rd original image

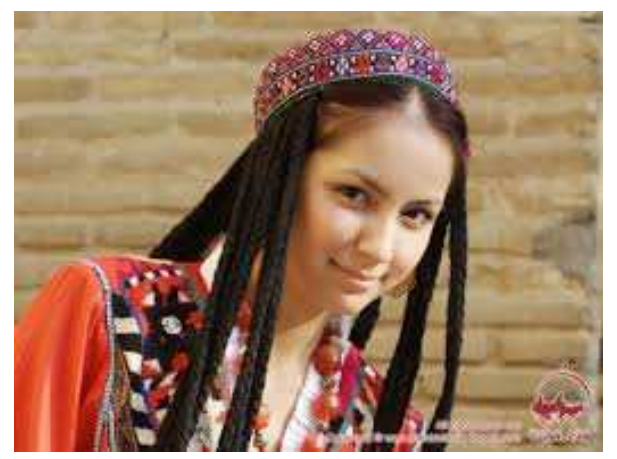

Figure 7: 4 th original image

Next, we need on the facial image, select the pixels with the characteristic color, to calculate the average intensity and standard deviation.

On the basis of knowledge about the values of the intensities of pixels and their possible variations is the image segmentation (Figure $8-11$ ).

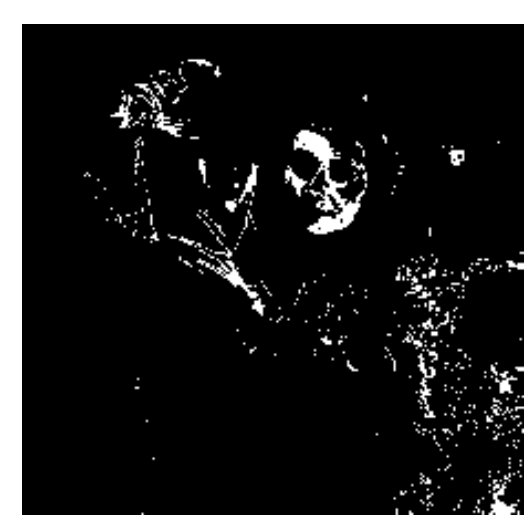

Figure 8: 1st Source Image After Segmentation

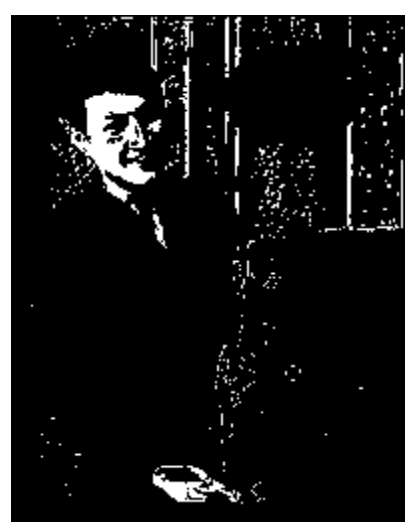

Figure 9: 2nd Source Image After Segmentation
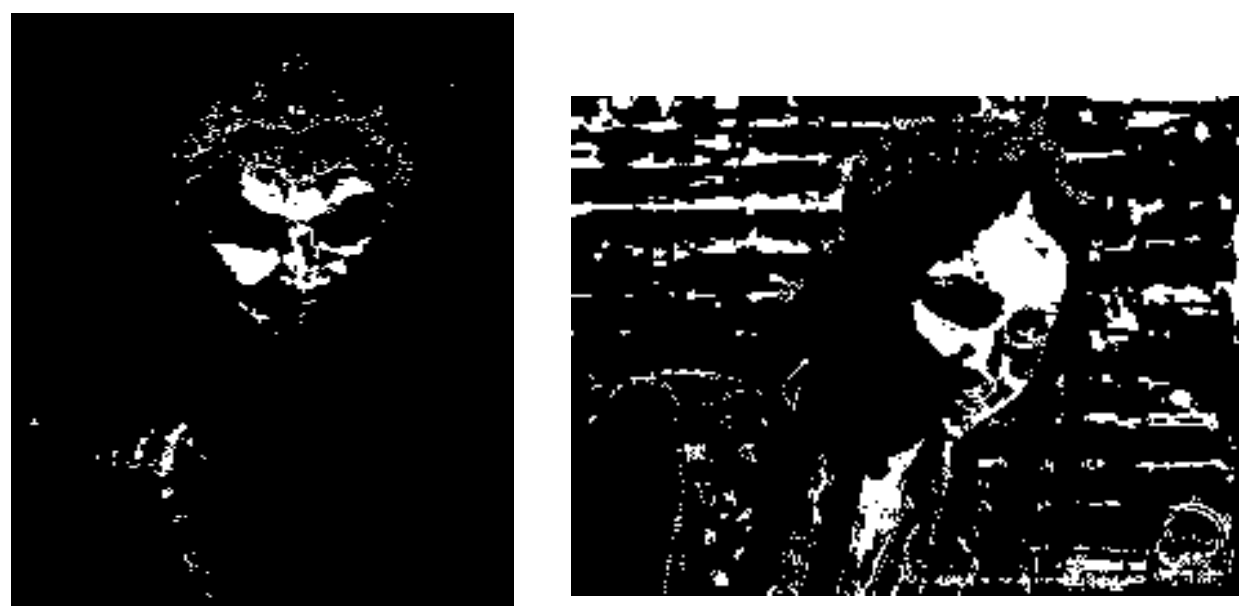

Figure 10: 3rd Source Image After Segmentation Figure 11: 4th Source Image After Segmentation

The aim of such segmentation is the selection of the face in the image. However, it is only natural that the image was present and other objects, the values of the intensities of pixels which coincided with the intensity of the pixels of the face. As a result, the segmented image except the face stand out and other objects. Now on the segmented image to find the 
image of the desired object, i.e. person. Search criteria may be different. This may be area, shape, etc. In this example, the criterion for the search face choose a square. On the basis of the previously selected segmentation method, we can assume that the person occupies the largest area. Therefore, the selection criteria for finding persons on the basis of the square will allow you to delete other objects that are smaller in area (Figure $12-15$ ).

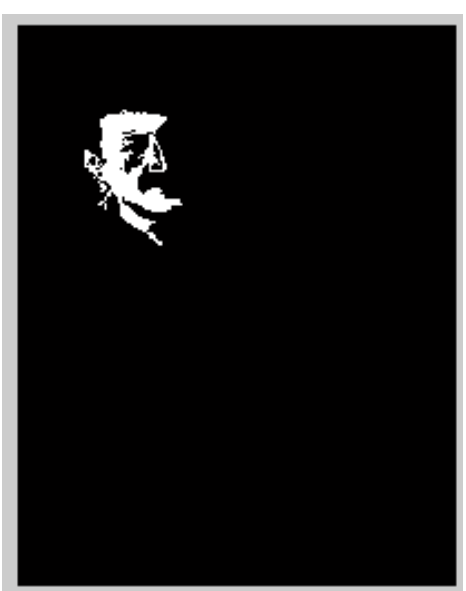

Figure 12: Original Image after Noise Removal

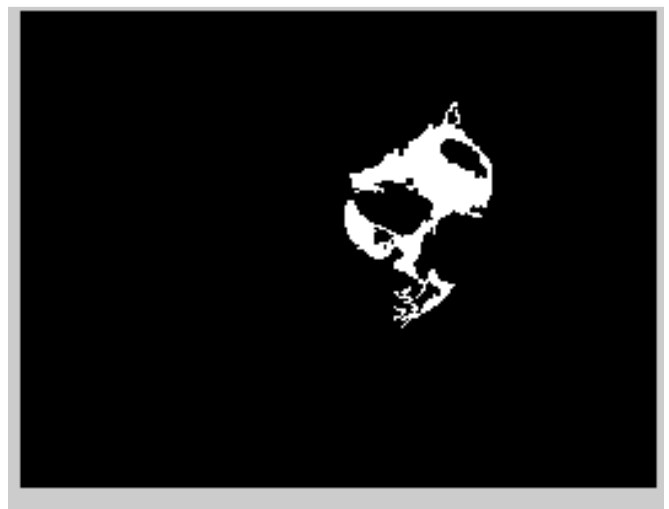

Figure 14: Original Image after Noise Removal

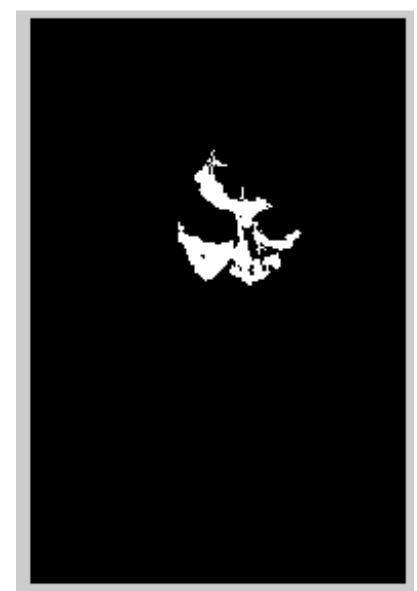

Figure 13: Original Image after Noise Removal

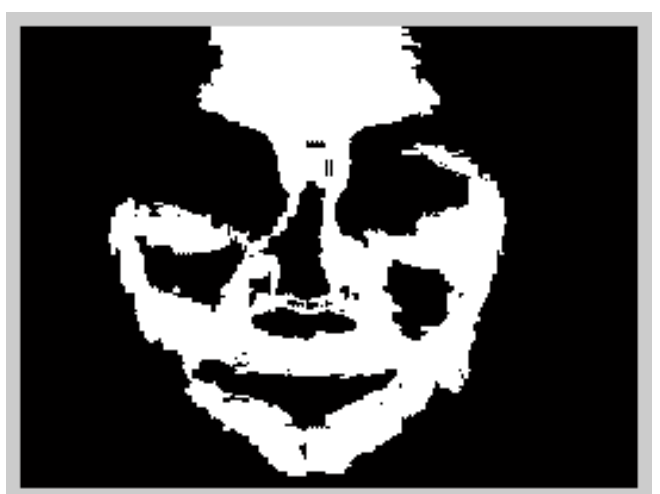

Figure 15: Original Image after Noise Removal

Remove other objects on the segmented face image (Figure 16-19).

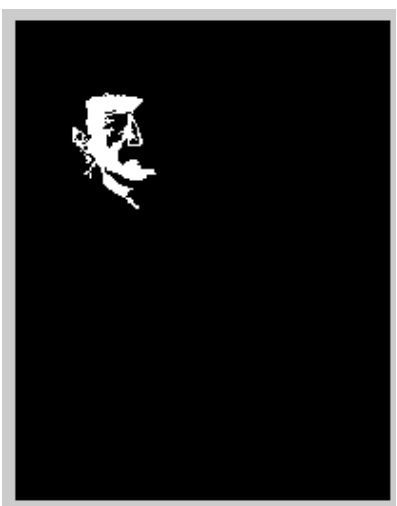

Figure 16: Original Image After Removal of Small Noise Objects

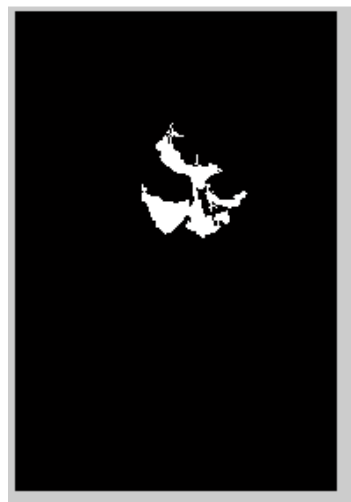

Figure 17: Original Image After Removal of Small Noise Objects 


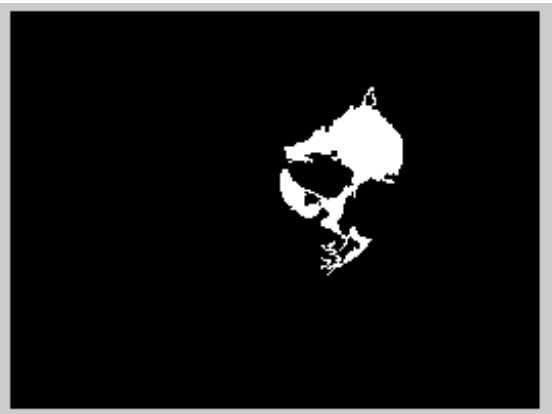

Figure 18: Original Image after Removal of Small Noise Objects

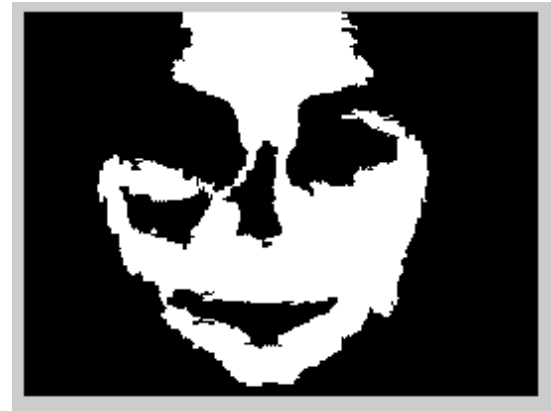

Figure 19: Original Image after Removal of Small Noise Objects

It should be noted that the chosen criteria may be insufficient for reliable determination of a search object, i.e., face image. This can occur if the image contains other objects with similar color, a person may be in the shade, slope, etc. it is Therefore necessary to use other criteria to search the image of the face. Such criteria can be based on a priori information about the shape of the face.

Thus, to improve the reliability recognition criterion search of the person in the image must be integrated.

Second, we perform the selection of the selected person, for example, a rectangle (Figure $20-23$ ).

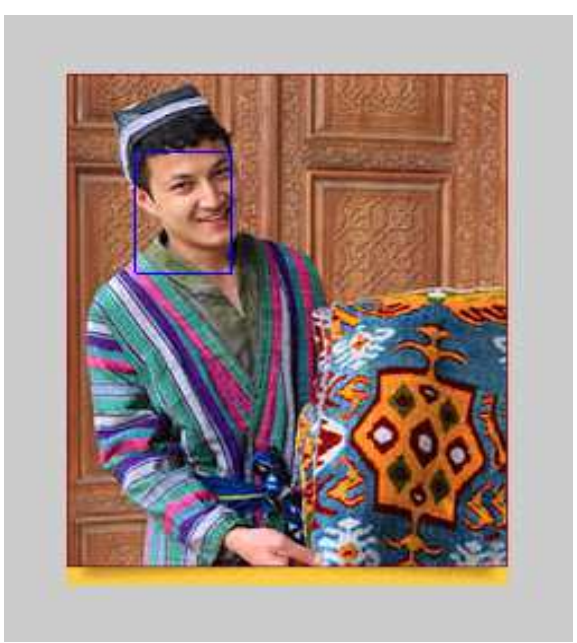

Figure 20: The Selected 1 - Face, By Rectangle

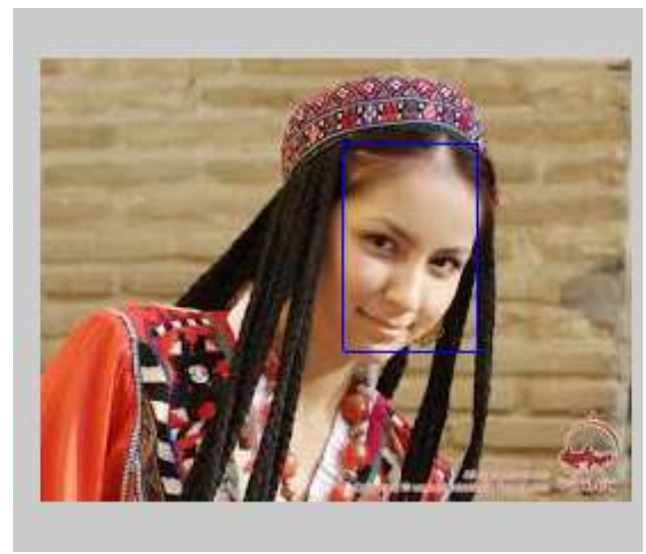

Figure 22: Selected 3 - Face, by Rectangle

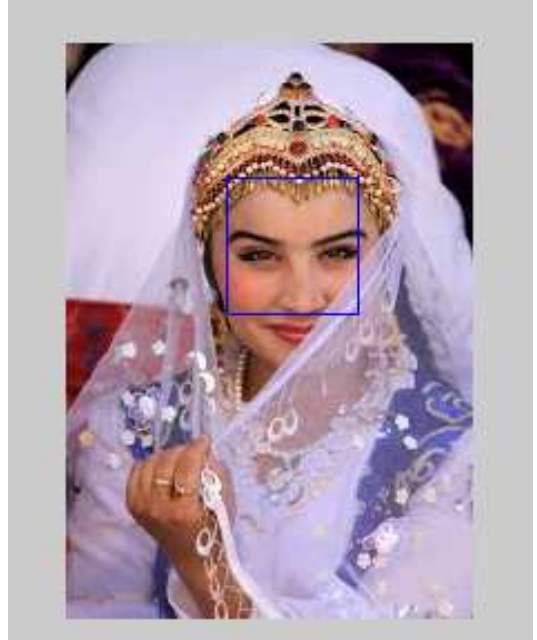

Figure 21: The Selected 2 - Face, By Rectangle

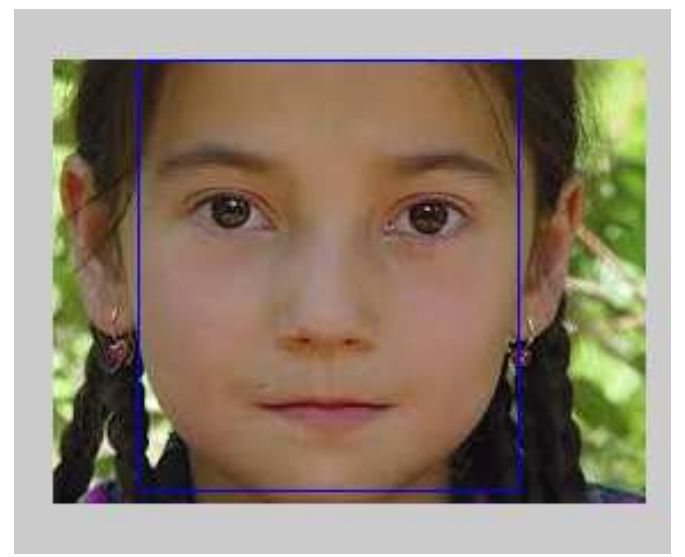

Figure 23: Selected 4-Person by Rectangle 
If an image is several persons at the same time, the considered above method must be modified. To discuss this in more detail. The first few steps (read the original image, the selection of characteristic pixels, the segmentation and filtering) the same as in the previous method, so we show only the results of these operations.

\section{CONCLUSIONS}

The paper presents fuzzy clustering algorithms for the space R, the method of image segmentation that use clustering algorithms. Advantages of this approach are:

- A Wide Range of Applications: Due to the choice of mappings and measures to adapt the algorithm for different tasks;

- Flexibility: Changing the threshold and the measure, it is possible to change the sensitivity of the algorithm;

- Speed: The algorithm works substantially faster algorithm for finding the boundaries of the method;

- Stability: The algorithm is more robust than methods based on finding borders, since the error, we lose not the entire region, and only a small portion of it.

\section{REFERENCES}

1. Pal S. K., King, R.A. (1981). Image enhancement using smoothing with fuzzy sets, IEEE Trans. Syst., Man and Cyberns., 11 (7), 494-501.

2. Mancuso M., Poluzzi R., Rizzotto G. A. (1994) Fuzzy filter for dynamic range reduction and contrast enhancement, Proc. IEEE Int. Conf. On Fuzzy Syst., IEEE Press, Piscataway, NJ, 264-267

3. Peli T., Lim J. (1982). Adaptive filtering for image enhancement, Optical Engineering, 21, 108-112.

4. Peng S., Lucke L. (1994). Fuzzy filtering for mixed noise removal during image processing, Proc. IEEE Int. Conf. On Fuzzy Syst., IEEE Press, Piscataway, NJ, 89-93.

5. Chi Z. Fuzzy algorithms: With Applications to Image Processing and Pattern Recognition. - London: Word Scientific, 1998. $225 p$.

6. Pal N.R., Pal S.K. (1993). A review of image segmentation techniques, Pattern Recognition, 26 (9), 1277-1294.

7. Sahoo P.K., Soltani S, Wong A.K., Chen Y.C. (1988). A survey of thresholding techniques, CVGIP, 41 (2), 233-260.

8. Pal S.K., King, R.A., Hishim A.A. (1983a). Automatic grey level thresholding through index of fuzziness and entropy, Patt. Recog. Lett. 1, 141-146.

9. Pal S.K., RosenfeldA. (1988). Image enhancement and thresholding by optimization of fuzzy compactness, Patt Recog. Lett, 7 , 77-86.

10. Pal S.K., Ghosh A. (1990). Index of area coverage of fuzzy subsets and object extraction, Patt. Recog. Lett., 11, 831-841.

11. Bhandari D., Pal N.R., Dutta Majumder D. (1992). Fuzzy divergence, probability measures of fuzzy events and image thresholding, Patt. Recog. Lett., 13, 857-867.

12. Boujemaa N., Stamon G., Lemoine J., Petit E. (1992a). Fuzzy ventricular endocardiogram detection with gradual focusing decision, Proc. IEEE Int. Conf. Of the Engineering in Medicine and Biology Society, 14, 1893-1894.

13. Gustafson E. E., Kessel W. (1979). Fuzzy clustering with a fuzzy covariance matrix, Proc. IEEE Conf. On Decision and 
Control, San Diego, IEEE Press, Piscataway, NJ, 761-766.

14. Jain, R., Kasturi R., Schunck B. G. (1995). Machine Vision, McGraw-Hill, NY.

15. Russo F., Ramponi G. (1994). Edge extraction by FIRE operators, Proc. IEEE Int. Conf. On Fuzzy Syst., IEEE Press, Piscataway, NJ, 249-253.

16. BezdekJ.C., ChandrasekarR., Attikiouzel Y.A. (1998a). A geometric approach to edge detection, IEEE Trans. Fuzzy Syst., 6 (1), 52-75.

17. Law T., Itoh H., Seki H. (1996). Image filtering, edge detection and edge tracing using fuzzy reasoning, IEEE Trans. Pattern Analysis and Machine Intelligence, 18, 481-491. 